\title{
EFFECT OF BODY MASS INDEX ON DYNAMIC BALANCE AND FUNCTIONAL ACTIVITY IN PERSONS WITH OSTEOARTHRITIS OF KNEE: A CROSS SECTIONAL OBSERVATIONAL STUDY
}

\author{
Dr. Mittal T. Raval ${ }^{1}$, Dr. Bhavna Gadhavi ${ }^{2}$ \\ ${ }^{1}$ Clinical Therapist M.P.T (Ortho), S.B.B College of Physiotherapy, V.S. Hospital Campus, Ellisbridge, Ahmedabad - \\ 380006. \\ ${ }^{2}$ Principal M.P.T (Ortho), Ahmedabad Institute of medical sciences, Near Karnavati Eye Hospital, Lapkaman,
} Ahmedabad- 380060.

\begin{abstract}
Osteoarthritis is a chronic and degenerative disease, with disease prevalence increasing with advancing age. Higher BMI is a significant risk factor for OA in weight bearing joints. Balance is an integral component of Activity of Daily Living. Obese individuals have more stable postural balance but may still experience difficulty in dynamic balance. Impairment in balance capacity raises the risk of falls for obese individuals. Impairment in components of mobility and balance affect functional activity of person.
\end{abstract}

Materials and Methods: 90 Knee osteoarthritis subjects that fulfill the inclusion and exclusion criteria and written informed consent was taken. Dynamic balance and functional activity were measured by SEBT score and WOMAC score respectively.

Results: The data was analyzed using Pearson correlation coefficient for correlation of SEBT and WOMAC with different BMI groups and ANOVA for comparison of SEBT and WOMAC within and between groups. It was found that there were no significant correlation of Body Mass Index(BMI) on dynamic balance and functional activity in persons with osteoarthritis of knee.

Conclusion: It is concluded that dynamic balance and functional activity in subjects with OA have no statistical significant difference in different groups of BMI. So, BMI does not have any correlation with dynamic balance in subjects with OA knee as well as BMI does not have any correlation with functional activity in subjects with OA knee. But, Clinically subjects with higher BMI shows reduced dynamic balance and functional activity compared with normal and overweight individuals.

Keywords: Osteoarthritis, Body Mass Index (BMI), Dynamic Balance, Star Excursion Balance Test, WOMAC (Western Ontario and MacMaster Universities Osteoarthritis Index).

\section{INTRODUCTION}

Osteoarthritis is a chronic and degenerative disease characterized by pain and gradual loss of joint cartilage. ${ }^{[1]}$ Knee osteoarthritis (OA) is one of the most prevalent musculoskeletal disorders worldwide. Primary osteoarthritis is mostly related to aging. It can present as localized, generalized or as erosive osteoarthritis. Secondary osteoarthritis is caused by another disease or condition. ${ }^{[2]}$

Individuals with knee OA suffer progressive loss of function, displaying increasing dependency in

${ }^{*}$ Corresponding author:

Email: mittalraval@ymail.com

http://dx.doi.org/10.20530/IJTA 34 1-6

ISSN 2320-138X @ 2016 walking, stair climbing and other lower extremity task. ${ }^{[3]}$ When OA affects weight-bearing joints, mainly the knee, it leads to a marked decline of muscle function and consequently to a reduction in balance and especially while performing sit to stand task and even gait alterations resulting in functional limitation and loss of independence. Therefore, OA is considered to be an intrinsic risk factor for the occurrence of fall. ${ }^{[3]}$ Eighty percent of patients develop medial compartment $\mathrm{OA}$ and they develop a varus or "bow - legged" deformity. Five to ten percent develop a lateral compartment OA of knee resulting in valgus or "knock - knee" deformity. ${ }^{[4]}$

The Body Mass Index is the most common method to quantify weight across a range of body sizes in adults. The BMI is an inexpensive and easy to use clinical measure that can be administered with minimal training. Higher BMI levels were associated 
with poorer mobility on performance based measure. ${ }^{[5]}$ These physical adaptations lead to impaired balance, abnormal gait patterns and increased incidence of muscle weakness - the top 3 risk factors for falls in older adults. ${ }^{[6]}$ The increased prevalence of obesity in older adults is especially concerning given the association between obesity and impaired physical function.

Balance is defined as the process that maintains the center of gravity within the body's base of support and requires constant adjustments that are provided by muscular activity and joint positioning. ${ }^{[1]}$ Balance function has been reported to decline with age, as evidenced by increased postural sway and decreased stability. ${ }^{[7]}$

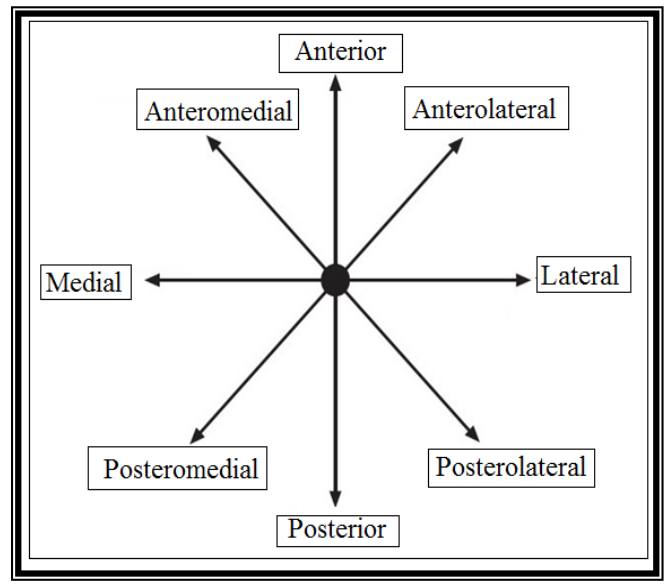

Figure 1: Star Excursion Balance Test

Limited researches have evaluated the impact of knee OA on dynamic balance and functional mobility. Most of previous studies utilized force platforms and sway - meter to measure postural sway and simple clinical tests like STEP TEST and TIME UP \& GO TEST to evaluate dynamic balance in OA patients. ${ }^{[3]}$

There are various methods to assess dynamic balance like $Y$ balance test, Balance error scoring system (BESS), The Star excursion balance test (SEBT) and The Modified Bass test, Time up and Go test (TUG). ${ }^{[8]}$ Star excursion balance test (SEBT) is one of the reliable methods to assess dynamic balance as it challenges a person's ability to maintain a stable base of support simultaneously performing reach outs. $^{[8]}$ It requires strength, flexibility and proprioception and lower extremity coordination. ${ }^{[9]}$ The SEBT is a dynamic postural control test that requires balance on 1 leg with maximum reach of the opposite limb. ${ }^{[10]}$
The Western Ontario and MacMaster Universities Osteoarthritis Index (WOMAC) evaluated knee pain and disability in the OA group. It is composed of the 3 subscales of perceived pain, stiffness and functional ability. ${ }^{[11]}$

Balance is an integral component of activities of daily living. Impairment in balance capacity raises the risk of falls. As a result of balance decrements, every day functioning may be impaired. Knee $O A$ is a factor that lowers the quality of life in the elderly. Therefore, it is necessary to conduct a study on the effect of BMI on dynamic balance and functional activity in persons with osteoarthritis of knee.

\section{METHODOLOGY}

The Study was approved by Institutional Ethical Committee.

\section{STUDY DESIGN : A Cross Sectional Study}

SOURCE OF DATA : This study was carried out at Various Orthopaedic Hospitals and Physiotherapy Departments of Ahmedabad.

\section{SAMPLE DESIGN : A Simple Random Sampling}

\section{STUDY DURATION : 9 Months}

SAMPLE SIZE ESTIMATION : The sample size was estimated on basis of data of pilot study. From pilot study SD for SEBT = 6.81 estimated sample size was 91 provides, $80 \%$ power assuming a significance level of $95 \%$, calculated with equation based on precision rate and confidence level. 90 Patients were taken for the study.

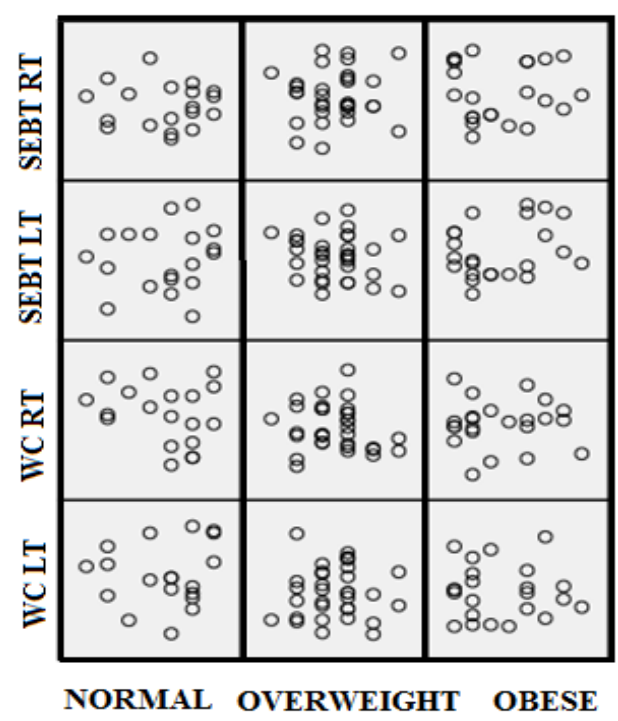

Figure 2 : Correlation of sebt and womac with different groups of BMI 
TABLE 1 : Comparison of BMI Groups with sebt and womac

\begin{tabular}{|c|c|c|c|c|}
\hline DIRECTION & \multicolumn{3}{|c|}{ BMI GROUP } & \multirow[t]{2}{*}{ p Value } \\
\hline Right Leg & $\frac{\text { Normal }}{(\text { Mean } \pm \text { SD })}$ & $\frac{\text { Overweight }}{(\text { Mean } \pm \text { SD) }}$ & $\begin{array}{c}\text { Obese } \\
(\text { Mean } \pm \mathrm{SD})\end{array}$ & \\
\hline Anterior & $59.22 \pm 8.14$ & $57.3 \pm 7.9$ & $56.5 \pm 9.4$ & 0.548 \\
\hline Anterolateral & $63.75 \pm 7.85$ & $61.9 \pm 7.5$ & $61.4 \pm 9.0$ & 0.574 \\
\hline Lateral & $63.33 \pm 7.04$ & $59.7 \pm 7.5$ & $60.7 \pm 8.5$ & 0.263 \\
\hline Posterolateral & $60.18 \pm 11.3$ & $58.8 \pm 7.35$ & $60.7 \pm 9.9$ & 0.675 \\
\hline Posterior & $47.15 \pm 11.6$ & $45.0 \pm 9.0$ & $44.8 \pm 9.1$ & 0.669 \\
\hline Posteromedial & $41.12 \pm 10.38$ & $39.5 \pm 10.9$ & $39.4 \pm 10.2$ & 0.852 \\
\hline Medial & $35.2 \pm 10.7$ & $32.6 \pm 9.6$ & $32.3 \pm 8.7$ & 0.548 \\
\hline Anteromedial & $57.3 \pm 7.9$ & $55.2 \pm 7.6$ & $53.5 \pm 9.7$ & 0.289 \\
\hline SEBT & $63.0 \pm 9.48$ & $61.7 \pm 8.5$ & $62.3 \pm 9.8$ & 0.860 \\
\hline WOMAC & $33.15 \pm 15.3$ & $34.5 \pm 15.4$ & $32.4 \pm 13.6$ & 0.847 \\
\hline \multicolumn{5}{|l|}{ Left Leg } \\
\hline Anterior & $58.0 \pm 5.64$ & $56.9 \pm 6.7$ & $58.8 \pm 7.9$ & 0.541 \\
\hline Anteromedial & $62.4 \pm 7.03$ & $61.6 \pm 7.6$ & $62.7 \pm 9.5$ & 0.893 \\
\hline Medial & $62.12 \pm 6.85$ & $60.6 \pm 7.0$ & $61.0 \pm 7.9$ & 0.741 \\
\hline Posteromedial & $60.53 \pm 11.4$ & $59.9 \pm 7.9$ & $61.2 \pm 9.0$ & 0.863 \\
\hline Posterior & $45.55 \pm 10.9$ & $46.11 \pm 8.72$ & $45.0 \pm 8.9$ & 0.906 \\
\hline Posterolateral & $41.19 \pm 11.3$ & $40.0 \pm 10.9$ & $41.2 \pm 9.7$ & 0.906 \\
\hline Lateral & $34.4 \pm 11.4$ & $32.7 \pm 10.7$ & $32.0 \pm 8.4$ & 0.715 \\
\hline Anterolateral & $56.7 \pm 7.9$ & $55.2 \pm 6.7$ & $54.5 \pm 7.4$ & 0.557 \\
\hline SEBT & $62.5 \pm 9.81$ & $62.0 \pm 8.4$ & $61.7 \pm 9.6$ & 0.912 \\
\hline WOMAC & $26.9 \pm 13.53$ & $37.2 \pm 16.4$ & $37.7 \pm 16.6$ & 0.061 \\
\hline
\end{tabular}

INCLUSION CRITERIA : Patients with clinical diagnosis of OA knee by orthopaedic surgeon, unilateral or bilateral OA knee, Age :- $40-65$ years, Both gender (male and female) were included. Grade 1 - 3 radiologically diagnosed OA knee according to Kellegren and Lawrence Grades of OA.

EXCLUSION CRITERIA : History of trauma or any musculoskeletal or neurological diseases affecting knee, hip or ankle except OA knee, Low back pain radiating to knee joint, Present complain of any ankle, foot or heel pain, $\mathrm{H} / \mathrm{o}$ Knee, hip and ankle surgery or intra articular corticosteroid injection, Any congenital abnormality around foot, ankle and toes, Any neurological disorders, cardiovascular problems, neoplastic condition problems. Vestibular Disorders with balance impairment, Ear infections.

OUTCOME MEASURES: 1. Modified WOMAC 2. Star Excursion Balance Scale

PROCEDURE

Patients were taken for the study with diagnosis of knee OA. Subjects willing to participate in the study were screened for inclusion and exclusion criteria. They were explained about the procedure written consent was taken. All the participant's height and weight measurement was taken and the BMI was calculated of each and every participants, according to WHO classification divided into three groups (Normal, Overweight, Obese). All the 


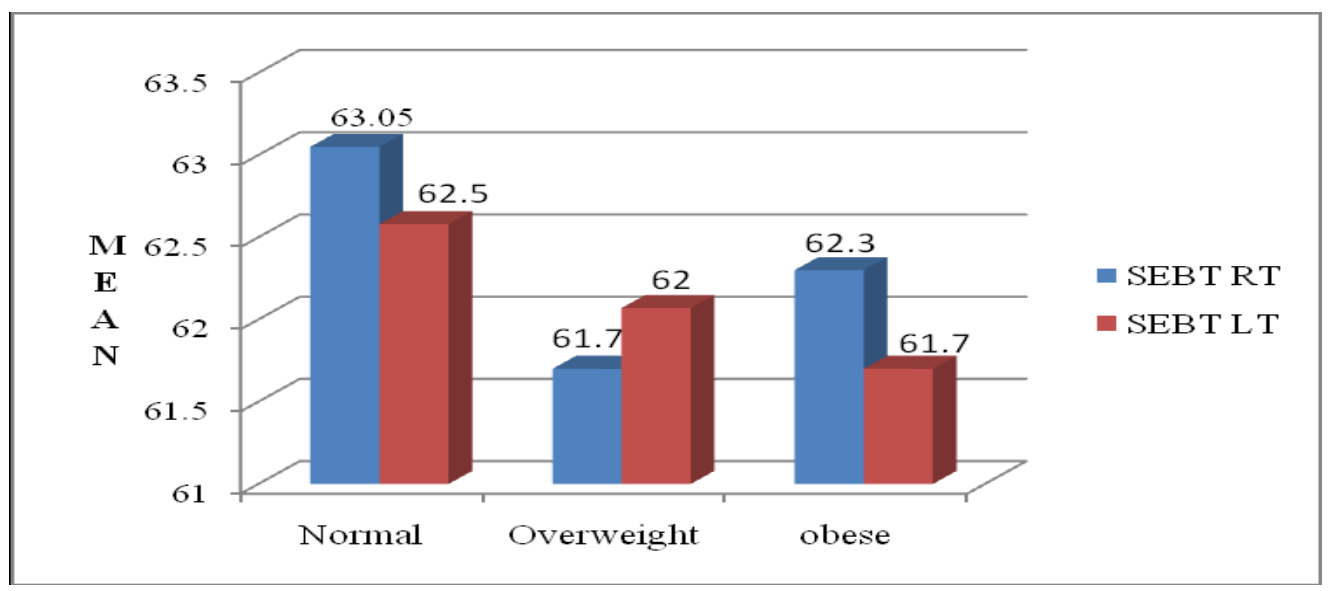

Figure 3 : Comparison of sebt with different groups of BMI

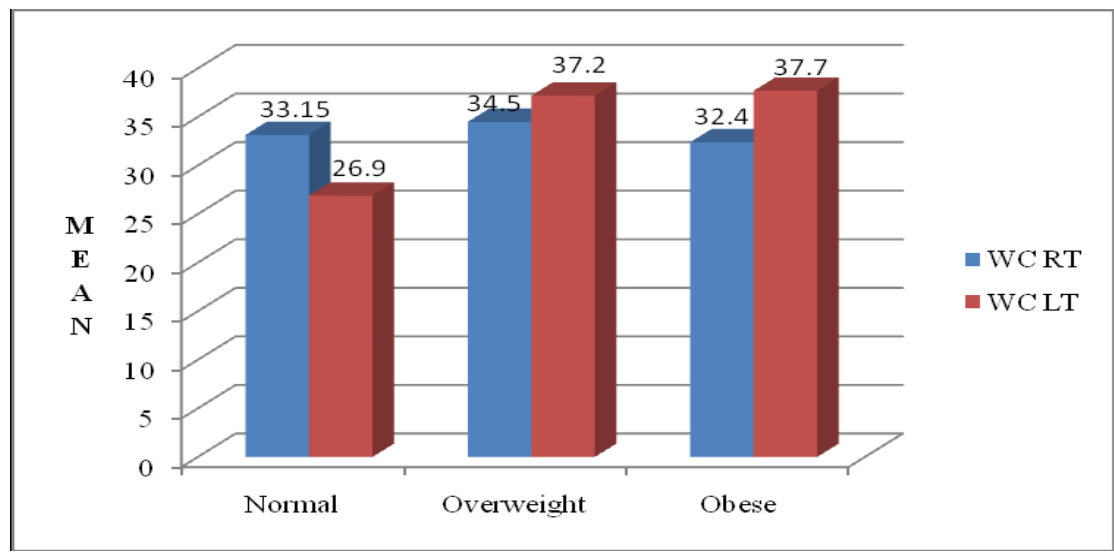

Figure 4 : Comparison of womac with different groups of BMI

individual's limb length was measured from ASIS to medial malleolus. This is used for calculating SEBT score.

The Star Excursion Balance Test (SEBT) is a dynamic postural control test that requires balance on 1 leg with maximum reach of the opposite limb. ${ }^{[14]}$ The SEBT was performed with the participants standing in the middle of the grid formed by eight lines extending out at $45^{\circ}$ apart from each other: anterior, posterior, medial, lateral, posterolateral, posteromedial, anterolateral, and anteromedial. The foot of tested limb was positioned in the center of grid of the star pattern. ${ }^{[31]}$ The subject is instructed to reach as far as possible, sequentially (either clockwise or counter clockwise), in all eight directions. Each Participant performed 8 reaches for 3 trials in a randomized order. The mean of 3 trials were taken for the 1 - way analysis of variance.
SEBT composite score was calculated by dividing the sum of maximum reached distance in anterior, anteromedial, anterolateral, medial, lateral, posterior, posteromedial, posterolateral directions by eight times the limb length of individual multiply by $100 .{ }^{[14]}$

SEBT Score $=\underline{A+A M+A L+M+L+P+P M+P L} * 100$

$$
8 * \text { Limb Length }
$$

\section{RESULT}

The Outcome measures were Dynamic Balance measured by Star Excursion Balance test and Functional Activity measured by Western Ontario and McMaster Universities Osteoarthritis Index (Modified WOMAC). Data analysis was done by using SPSS software version 20 and Microsoft Excel 2007. A significant level was set at the $95 \%$ confidence interval for all statistical parameters $(p<0.05)$. 
Pearson Correlation test was used as the data was found to be normally distributed. It is used to measure correlation of SEBT and WOMAC with different BMI Groups. ANOVA was used to measure Comparison of SEBT and WOMAC with different BMI Groups \& Comparison of SEBT and WOMAC within and between Groups. Using ANOVA we determined that there was no statistically significant difference in Star Excursion Balance Score and WOMAC with BMI. It was found that there was clinically significant difference between normal and obese groups. There was also significant difference between normal and overweight groups.

An ANOVA was used to compare the Star Excursion Balance Score between the three groups. (Group 1 - Normal individuals), (Group 2 Overweight individuals), (Group 3 - obese individuals). The result shows no statistically significant difference between BMI group as well as within BMI group as $\mathrm{p}$ value $>0.05$ indicating there was no effect of BMI on Dynamic balance (SEBT) and Functional activity(WOMAC) in OA Knee patients.

\section{DISCUSSION}

Osteoarthritis is the most common musculoskeletal disorder and leading cause of pain and disability in the community. The symptoms of Knee OA affect the activities of daily living which consequently may lead to limitation of one's independence and affect health related quality of life. Star Excursion Balance Test is dynamic balance screening test which is used in various fields of physiotherapy like neurological, orthopaedic, sports and rehabilitation programs. ${ }^{[14]}$

Pearson Correlation test was used to analyze correlation between SEBT in all directions and WOMAC in participants with normal BMI. The result of the study indicated that there was negative correlation between SEBT and WOMAC in subjects with normal BMI for right leg and positive correlation for left leg. Hrysomallis (2011) in his review paper reported that in SEBT, other variables such as power, flexibility and coordination were also effective beside balance. So, he concluded that change in BMI was not only the factor altering dynamic balance.

The Pearson Correlation of SEBT and WOMAC with overweight group of BMI showed positive correlation with SEBT and negative correlation with WOMAC for right leg. In left leg, there was negative correlation of WOMAC and SEBT in all directions except lateral direction. Lateral direction showed positive correlation for left leg. Hergenroeder et al. (2011) research findings who did not report any significant relationship between weight and balance.

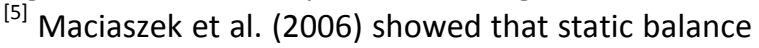
has a relationship with weight but dynamic balance does not have. One reason for this can be explained because difference between age, gender and the level of activity of the subjects. ${ }^{[13]}$

The Pearson Correlation of SEBT and WOMAC with obese group of BMI showed negative correlation between SEBT and WOMAC for right leg and positive correlation of SEBT in all directions and negative correlation of WOMAC in left leg. Higher BMI levels were associated with poorer mobility but not balance. ${ }^{[5]}$

An ANOVA test was done to compare WOMAC and the three BMI groups mean values. As the result of the study showed there was no statistical difference between the BMI groups with SEBT and WOMAC and within the group also with SEBT and WOMAC. But, it showed clinically significant difference between normal and obese groups. It also showed difference between normal and overweight groups, which supports Kumaresan A 2012 that Higher Body Mass Index associated with poorer balance. Study showed that Higher Body Mass Index showed lesser amount of SEBT score compare to overweight and Normal individuals.

Obese individuals show lesser centre of mass displacements and slower center of mass velocity than normal Body Mass Index following balance test performances. ${ }^{[14]}$ An accurate evaluation of balance control in knee OA patients is necessary before further treatment can be recommended or developed. Since all patients with central nervous system or vestibular organ abnormalities had been excluded, we can conclude that the decrease in balance control of the moderate to severe group was due to musculoskeletal fatigue, and a decline in motor functions of the spine and lower extremities.

Therefore, this study indicates that not only treatment for $\mathrm{OA}$ but also training to improve balance is necessary in patients with progressive knee OA. ${ }^{[15]}$ Although increased BMI in older adults may influence balance, other factors associated with aging also may contribute to postural instability. These factors include sarcopenia, defined as the agerelated loss of skeletal muscle mass and strength (force-generating capacity), changes in body fat distribution, specifically an increase in visceral 
abdominal fat and a decrease in subcutaneous fat; and a decline in the quality of skeletal muscle.

It is plausible that changes in skeletal muscle and body fat distribution may be related more to postural instability than to BMI alone, which may explain the lack of a stronger relationship between balance and BMI in the current study. Obesity is associated with increased burden of chronic disease and decreased physical activity level, both of which have been shown to negatively affect mobility. Study showed that Obese individuals were more likely to have less physical activity compared with normal and overweight individuals.

\section{CONCLUSION}

It is concluded that dynamic balance and functional activity in subjects with $O A$ have no statistical significant difference in different groups of BMI. But, clinically subjects with higher BMI showed reduced dynamic balance and functional activity compared with normal and overweight individuals.

Future study can be done by bifurcating the subjects according to the stages of OA knee.

\section{ACKNOWLEDGEMENT}

I would like to thank my parents, my brother \& sister who have always encouraging me. My special thanks to my guide Dr. Bhavna gadhavi for her cooperation, constant encouragement.

\section{REFERENCES}

1) Greve J, Carolina A et al. Correlation between body mass index and postural balance, Clinical science, 2007. Clinics Vol. 62.

2) Mahajan A, Verma S, Tandon V. Osteoarthritis JAPI 2005 ; Vol. 53.

3) Shaheen A, Ayad K, Impact of Chronic Osteoarthritis of Knee Joint on Postural Stability and Functional Mobility in Women, Department of orthopaedic, Faculty of Physical Therapy, Cairo University. 2008 Jan. Vol. 13 (1).

4) Brotzman S B , Wilk K E, Clinical Orthopaedic Rehabilitation: An Evidence Based Approach , $2^{\text {nd }}$ edition, United States of America, Mosby ; 2003.

5) Hergenroeder AL, Wert DM, Hile ES, Studenski SA, Brach JS. Association of Body Mass Index With SelfReport and Performance-Based Measures of Balance and Mobility. Physical Therapy. 2011 Jun 16;91(8):1223-34. Available from: http://dx.doi.org/10.2522/ptj.20100214.
6) Smith D R, Pechak C M et al. Biomechanical Effects of Obesity on Balance, International Journal of Exercise Science, 2012; Vol. 5 (4): pg $301-320$.

7) Kisner C, Nichols D et al. Static and Dynamic Balance Responses in Persons with Bilateral Knee Osteoarthriis, Journal of Orthopaedic \& Sports Physical Therapy. 1997 January; Vol. 25 (1).

8) Bhat R, Moiz J A, Comparison of Dynamic Balance in Collegiate Field Hockey and Football Players Using Star Excursion Balance Test, Asian Journal of Sports Medicine, 2013 September; Vol. 4 (3) : pg 221-229.

9) Schuddinck D, Lowe R, Star Excursion Balance Test Physiopedia [Internet], Available from: "http://www.physiopedia.com/Star Excursion Balan ce Test".

10) Bouillon LE, Baker JL. Dynamic Balance Differences as Measured by the Star Excursion Balance Test Between Adult-aged and Middle-aged Women. Sports Health. 2011 Sep;3(5):466-9. Available from: http://dx.doi.org/10.1177/1941738111414127.

11) Felson D T, Misra $D$ A et al. Measures of Knee Function, Arthritis Care and Research, 2011 November; Vol.63.

12) Miller T, NSCA's Guide and Assessments, National strength and conditioning Association, 2012.

13) Bakhtiari R A, Evaluation of Static and Dynamic Balance and Knee Proprioception in Young Professional Soccer Players, Annals of Biological Research, 2012, Vol.3 (6): pg 2867-2873.

14) Kumaresan A, Kavithayini $T$, The impact of Body Mass Index on Performance of the Star Excursion Balance Test, International Journal of Pharmaceutical Science and Health care, 2012 April; Vol. 2 (2).

15) Kim H-S, Yun DH, Yoo SD, Kim DH, Jeong YS, Yun J-S, et al. Balance Control and Knee Osteoarthritis Severity. Annals of Rehabilitation Medicine. 2011;35(5):701. Available from: http://dx.doi.org/10.5535/arm.2011.35.5.701 